\title{
Psychological effect of COVID-19 pandemic on healthcare professionals of Yemen and coping strategies
}

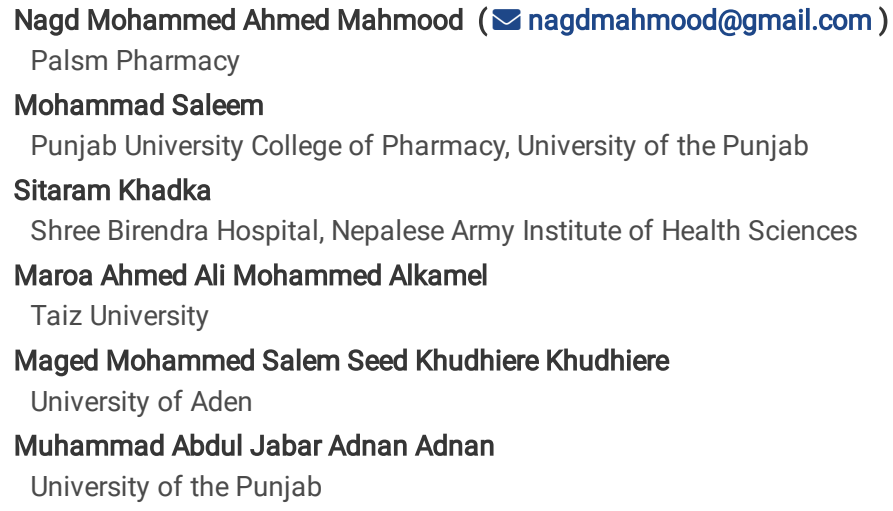

Research Article

Keywords: Depression, Pandemics, Yemen, COVID-19, Anxiety Disorders, Adaptation, Psychological

Posted Date: April 19th, 2021

DOI: https://doi.org/10.21203/rs.3.rs-393255/v1

License: (c) (1) This work is licensed under a Creative Commons Attribution 4.0 International License. Read Full License 


\section{Abstract}

Background: COVID-19 pandemic has triggered psychological stress such as anxiety and depression among people around the globe. Due to the nature of the job, healthcare professionals are at high risk of infection and are facing social stigma as well. In such a scenarios, it has aggravated their mental health and they are applying various measures to cope with such adverse scenario. Therefore, this research is conducted with the objective to evaluate the psychological influence of the COVID-19 pandemic among healthcare professionals of Yemen and the coping strategies adopted thereof.

Methods: A web-based, as well as face-to-face cross-sectional study, was carried out from July 2020 to December 2020 among healthcare professionals currently working in different provinces of Yemen using a standard questionnaire. The generalized anxiety disorder scale (GAD-7), patient health questionnaire (PHQ-9), and Brief-COPE scales were applied for evaluation of anxiety, depression, and the coping strategies among them.

Results: A total of 197 healthcare professionals ( $N=197)$ participated in the study where the majority were male $68.5 \%(n=135)$ in gender and physicians $42.13 \%(n=83)$ by profession. The prevalence of both anxiety $(6.84 \pm 5.67$ for male and $7.37 \pm 4.44$ for female) and depression $(8.06 \pm 6.51$ for male and $9.56 \pm 6.46$ for female) were found of mild category among the respondents. A significant statistical difference was observed between physician versus nurse regarding anxiety and depression based on the working area $(p=0.017)$. Trained professionals demonstrated less anxiety $(6.29 \pm 5.33)$ and depression $(7.90 \pm 6.78)$ as compared to untrained ones. Source of stress varied in a different province where high fear of self-health and family members was found more in female (3.90 \pm 1.00$)$ and such fear was found more in province Sanaa, Lahij, and Abyan (4.75 \pm 0.96$),(4.57 \pm 1.27)(4.50 \pm 2.12)$ respectively. Religion was indicated as a highly adopted coping strategy meanwhile emotional support was found the least used.

Conclusions: COVID-19 pandemic has aggravated the psychological stress among healthcare professionals of Yemen. Our findings illustrate the requirement of efficient policies through administrative, clinical, and welfare perspectives from the regulatory body in preparedness and preventive measures towards such pandemic that aids healthcare professionals to provide service in a stress-free condition and thus better healthcare delivery are assured.

\section{Background}

The COVID-19 pandemic has become a serious public health threat worldwide. The World Health Organization (WHO), on 30 January 2020, announced the occurrence of the novel coronavirus and declared a Public Health Emergency of International Concern (PHEIC) under the International Health Regulations (IHR) [1]. Later, it was declared a pandemic on 11 March 2020 [2].

In such a critical situation, life has been changed due to the restrictions of movement and social contacts. In fact, healthcare professionals (HCPs) wholeheartedly continued providing service with a high risk of getting infected with COVID-19 in such a grave situation. Therefore, HCPs are regarded as one of the most susceptible types of professionals to get psychological problems and mental catastrophe amid COVID-19 pandemic [3]. Different studies are consistently showing that the HCPs experience more stress related to work as compared to the general public [4].

As a standard procedure of containment strategy in such pandemics, a lockdown approach is usually imposed to bound the disease spread and lessen new potential cases by maintaining social distancing [5]. Though it is somewhat feasible for general people to assume such measures, the HCPs are, by the nature of their profession, left exposed to deal with the health-related issues that arise due to such situation and they have to be exposed to the situation. HCPs experience unexpectedly lengthy office hours as they have to deal with a load of cases due to such pandemic with the available resources and infrastructure that may not be up to the standard in such an emergency [6]. They often face physical distress and from time to time difficulties in breathing while wearing personal protective equipment (PPE), which is mandatory for safety measures to get saved from viral exposure [7].

Because little is known about the COVID-19, and subsequently, without the proven therapy, many HCPs are unrehearsed to perform duties [8]. The fear of autoinoculation, social stigma, and the risk of transmitting the disease to the family members and friends are adding extra burden to them that definitely impact their mental health [8-10].

Thus, it is particularly significant to assess the HCPs who are at greater risk of exhaustion and are probable to get suffered from anxiety, depression, and stress in such a pandemic. It is also equally essential to recognize and manage the responsible factors for such mental stress. The mental health status of HCPs of Yemen and their coping strategies have not yet been fully studied. Therefore, this study is conducted with the objectives to assess the anxiety and depression faced by Yemeni HCPs and to determine the coping strategies implemented amid COVID-19 pandemic.

\section{Methods}

\section{Study design and participants}

A cross-sectional survey was carried out via Google form as well as a self-administered questionnaire among healthcare professionals face-to-face for those who were not reachable with web-based survey in Yemen from July 2020 to December 2020. A total of 197 participants were selected by convenience sampling from different provinces all over Yemen.

The survey included questions regarding psychological screening, bothering issues faced by the health professionals, assessment of sources of the distress, coping strategies, or behavior. The questionnaire was developed and distributed in English and Arabic language. The questionnaire was validated by two experts in the field and was pilot tested among five participants.

\section{Study site}


The study was conducted in Yemen which is a country with a low-income economy and the poverty and the decade-long civil war has seriously affected the country. Many healthcare substructures are vulnerable and the basic healthcare facility is unobtainable to many people. Moreover, the shortage of medicines and medical equipment, the fragile healthcare status, and the limited healthcare resources are the challenging factors for the healthcare delivery amid COVID19 pandemic in Yemen [11].

\section{Inclusion/Exclusion criteria}

All the healthcare professionals comprising physicians, pharmacists, nurses, and others who provided care at the medical center were included in this study. The professionals who refused to participate, from a non-healthcare profession and those who cannot read and write were excluded from the study.

\section{Study questionnaire}

The study questionnaire was categorized into four sections. All the questions were in understandable language and the participant was required to answer the questions on their own.

The first section of the questionnaires was about the demographics of participants that provided personal and organizational information of the health employee.

The second section of the questionnaire comprised of total of seven questions linked to the generalized anxiety scale (GAD-7) for anxiety assessment [12]. It had seven items with a score 0 (not at all) to 3 (nearly every day) that provided a 0 to 21 score. The total score was classified into four severity groups, namely; minimal to none ( $\leq 4)$, mild (5-9), moderate (10-14), and severe $(\geq 15)$.

The third section consisted of total of nine questions related to patient health questionnaire (PHQ-9) to assess depression [13]. It had nine items with a score 0 (not at all) to 3 (nearly every day) that provided a 0-27 score. The total score was divided into five severity groups, namely; minimal to none ( $\leq 4)$, mild (5- 9), moderate (10-14), moderately severe (15-19), and severe ( $\geq 20$ ) [8]. In the current study, the participants' attaining score $\geq 10$ on GAD-7 and PHQ-9 were regarded as anxiety and depression, respectively.

The sources of distress from the current pandemic were measured with a 14-item scale designed from an earlier study on anxiety among university students amid SARS outbreak [14]. It was based on a two-point Likert-scale. The items were categorized under 4 scales such as the health of self, family, and loved ones (possible score: 3 to 6); transmission (possible score: 3 to 6); containment (possible score: 3 to 6 ); measures taken by authority (possible score: 3 to 6); and effects on daily activities (possible score: 3 to 6 ).

The fourth section was all about 28-questions of the Brief-COPE scale [15]. It aimed to identify the coping strategies implemented amid COVID-19 pandemic. It consisted of four response choices ranging from the importance of doing activities to cope with the outbreak; (a) not doing this at all, (b) a little bit, (c) moderate amount, (d) doing this a lot. That scale was developed to discover the 14 coping methods: self-distraction, active coping, denial, and substance use, use of emotional support, venting, behavioral disengagement, acceptance, positive reframing, planning, humor, use of instrumental support, religion, and selfblame. Likely scores for every subscale were in a range of 2 to 8 , where higher scores indicated propensity to appliance the analogous coping style.

\section{Ethical approval}

The study has been reviewed and approved by The Human Ethical Committee, University of the Punjab, Lahore. The ethical and professional considerations were followed throughout the study to keep the data and investigational information strictly confidential.

\section{Statistical analysis}

The data were coded, entered, and analyzed from SPSS (IBM, version 22). Results were articulated by using descriptive statistics where continuous data were expressed as mean and standard deviation (SD) whereas categorical data were presented as numbers and percentages. A p-value of less than 0.05 was considered statistically significant.

Independent t-test and ANOVA test were executed, wherever applicable, for comparison of the difference of scores related to anxiety, depression, source of distress, and coping strategies among demographic variables. Furthermore, for trior polychotomus variables, a series of post-hoc analysis with Bonferroni adjustment was implemented to evaluate significance among intergroup variables.

\section{Results}

There was a total of 197 respondents $(n=197)$ in our study. The demographic of respondents with anxiety and depression scores are depicted in table 1 . The majority of the participants were of age between 20-30 years $(n=111)$ followed by 30-40 years $(n=44)$ and $\geq 40$ ( $n=42)$. Most of the participants were male (68.5\%) and almost half of the respondents were physicians $(n=83)$ followed by other health professionals $(n=41)$, pharmacists $(n=37)$, and nurses $(n=36)$. Approximately $21 \%$ of total HCPs were working in COVID-19 isolation wards and $11 \%$ were performing duties in COVID- 19 intensive care unit (ICU). Meanwhile, $13.7 \%$ of respondents were working in quarantines, and the rest of the $53.3 \%$ were in other healthcare areas.

Table 1 Demographics with Anxiety and Depression Score 


\begin{tabular}{|c|c|c|c|}
\hline Variables & $N(\%)$ & $\begin{array}{l}\text { Anxiety Score } \\
\text { (Mean } \pm S D)\end{array}$ & $\begin{array}{l}\text { Depression Score } \\
\text { (Mean } \pm \text { SD) }\end{array}$ \\
\hline \multicolumn{4}{|l|}{ Age (Years) } \\
\hline $20-30$ & $111(56.34)$ & $6.74 \pm 5.37$ & $8.73 \pm 6.57$ \\
\hline $30-40$ & $44(22.33)$ & $7.50 \pm 4.93$ & $8.79 \pm 6.80$ \\
\hline$>40$ & $42(21.32)$ & $7.21 \pm 5.57$ & $7.73 \pm 6.16$ \\
\hline \multicolumn{4}{|l|}{ Gender } \\
\hline Male & $135(68.53)$ & $6.84 \pm 5.67$ & $8.06 \pm 6.51$ \\
\hline Female & $62(31.47)$ & $7.37 \pm 4.44$ & $9.56 \pm 6.46$ \\
\hline \multicolumn{4}{|l|}{ Occupation } \\
\hline Physician & $83(42.13)$ & $7.02 \pm 5.30$ & $7.40 \pm 6.11$ \\
\hline Nurse & 37 (18.78) & $7.38 \pm 4.54$ & $11.21 \pm 6.99$ \\
\hline Pharmacist & $36(18.27)$ & $6.33 \pm 5.44$ & $9.15 \pm 7.21$ \\
\hline Other HCPs & $41(20.81)$ & $7.24 \pm 5.94$ & $8.53 \pm 6.52$ \\
\hline \multicolumn{4}{|c|}{ Experience (years) } \\
\hline$<5$ & $110(55.84)$ & $6.69 \pm 5.33$ & $8.16 \pm 6.21$ \\
\hline $5-10$ & $31(15.74)$ & $7.45 \pm 5.30$ & $9.97 \pm 7.11$ \\
\hline$>10$ & $56(28.43)$ & $7.41 \pm 5.30$ & $8.46 \pm 6.52$ \\
\hline \multicolumn{4}{|l|}{ Province } \\
\hline Aden & $44(22.33)$ & $6.66 \pm 5.36$ & $7.84 \pm 5.67$ \\
\hline Hadramaut & $52(26.39)$ & $7.30 \pm 5.62$ & $8.55 \pm 6.64$ \\
\hline Taiza & 59 (29.95) & $8.05 \pm 4.66$ & $10.39 \pm 6.38$ \\
\hline Aldhaleh & $9(4.57)$ & $3.11 \pm 5.23$ & $3.78 \pm 6.22$ \\
\hline Sanaa & $4(2.03)$ & $6.75 \pm 4.64$ & $6.75 \pm 4.03$ \\
\hline $\mathrm{lbb}$ & $8(4.06)$ & $8.12 \pm 2.85$ & $9.25 \pm 5.33$ \\
\hline Abyan & $2(1.01)$ & $1.5 \pm 0.70$ & $1 \pm 1.41$ \\
\hline Hajjah & $1(0.51)$ & 2.00 & 6.00 \\
\hline Lahij & $11(5.58)$ & $4.29 \pm 4.71$ & $4.57 \pm 5.69$ \\
\hline Shabwah & $7(3.55)$ & $7.09 \pm 7.54$ & $9.36 \pm 9.51$ \\
\hline \multicolumn{4}{|c|}{ Working area (Placement) } \\
\hline Quarantine & $27(13.70)$ & $6.30 \pm 5.72$ & $8.30 \pm 5.91$ \\
\hline Isolation & $43(21.83)$ & $7.25 \pm 4.02$ & $7.67 \pm 5.94$ \\
\hline ICU & $22(11.17)$ & $7.40 \pm 5.88$ & $11.73 \pm 7.32$ \\
\hline Other & 105 (53.30) & $7.01 \pm 5.80$ & $8.28 \pm 6.61$ \\
\hline \multicolumn{4}{|c|}{ Training (COVID-19) } \\
\hline Yes & $116(58.88)$ & $6.29 \pm 5.33$ & $7.90 \pm 6.78$ \\
\hline No & 81 (41.12) & $8.04 \pm 5.12$ & $9.43 \pm 6.06$ \\
\hline
\end{tabular}


The mean anxiety level for males $(6.84 \pm 5.67)$ and females $(7.37 \pm 4.44)$ was found to be of mild category. Similarly, the depression was also rated as mild type in both genders (male $=8.06 \pm 6.51$, female $=9.56 \pm 6.46$ ). Out of total respondents, $116 \mathrm{HCPs}$ who were trained regarding COVID-19 management had shown less anxiety $(6.29 \pm 5.33)$ as compared to the non-trained (8.04 \pm 5.12$)$ ones. Similarly, depression score of trained HCPs was found less (7.90 \pm 6.78$)$ as compared to untrained $(9.43 \pm 6.06)$ ones.

The comparison of scores related to anxiety and depression in the categories of age, occupation, experience, posting, and training are shown in table 2. The anxiety and depression score for all age groups were not statistically significant, meanwhile, significant statistical differences of depression score were reported in occupation wise comparison between physician versus nurse $(p=0.017)$.

Table 2 Post-Hoc Analysis (Bonferroni Correction for Anxiety and Depression Scores in Categories Age, Occupation, Experience, Posting, and Training)

\begin{tabular}{|c|c|c|}
\hline Variables & $\begin{array}{l}\text { Anxiety Score } \\
\text { (P-value) }\end{array}$ & $\begin{array}{l}\text { Depression Score } \\
\text { (P-value) }\end{array}$ \\
\hline \multicolumn{3}{|l|}{ Age (Years) } \\
\hline $20-30$ Vs $30-40$ & 1.00 & 1.00 \\
\hline $20-30 \mathrm{Vs}>40$ & 1.00 & 1.00 \\
\hline $30-40$ Vs $>40$ & 1.00 & 1.00 \\
\hline \multicolumn{3}{|l|}{ Occupation } \\
\hline Physician Vs Nurse & 1.000 & 0.017 \\
\hline Physician Vs Pharmacist & 1.000 & 1.000 \\
\hline Physician Vs others & 1.000 & 0.926 \\
\hline Nurse Vs Pharmacist & 1.000 & 0.119 \\
\hline Nurse Vs Others & 1.000 & 0.935 \\
\hline Pharmacist Vs Others & 1.000 & 1.000 \\
\hline \multicolumn{3}{|l|}{ Experience } \\
\hline$<5$ Vs $5-10$ & 1.000 & 0.526 \\
\hline$<5 \mathrm{Vs}>10$ & 1.000 & 1.000 \\
\hline $5-10 V s>10$ & 1.000 & 0.913 \\
\hline \multicolumn{3}{|l|}{ Working area (Posting) } \\
\hline Quarantine Vs Isolation & 1.000 & 1.000 \\
\hline Quarantine Vs ICU & 1.000 & 0.397 \\
\hline Quarantine Vs Others & 1.000 & 1.000 \\
\hline Isolation Vs ICU & 1.000 & 0.106 \\
\hline Isolation Vs Others & 1.000 & 1.000 \\
\hline ICU Vs Others & 1.000 & 0.144 \\
\hline
\end{tabular}

The source of distress was demonstrated in table 3. The fear of health of self and family members was found more in females as a source of distress $3.90 \pm 1.00$. Such fear as a source of distress was found higher in the provinces Sanaa (4.75 \pm 0.96$)$, Lahij (4.57 \pm 1.27$)$, and Abyan (4.50 \pm 2.12$)$ as compared to other provinces. The higher level of source of distress was reported as an effect on daily activity in province number Hajjah (6.00). The transmission source of distress was highest in province Lahij $(5.00 \pm 1.00)$ followed by province Aldhaleh (4.67 \pm 1.41$)$ meanwhile lowest was of province Hajjah (3.00). Containment was reported highest in province Shabwah (5.27 \pm 3.38$)$ and in HCPs who have experienced between 5-10 years (5.10 \pm 2.10$)$ and the maximum between age variable between $30-40(5.04 \pm 1.80)$. However, the measures taken by the authority were highest in province $8(4.50 \pm 2.12)$ and HCPs who worked in ICU $(4.00 \pm 1.34)$.

Table 3 Source of Distress among Respondents 


\begin{tabular}{|c|c|c|c|c|c|}
\hline Variables & Health of self/family/loved-ones & Transmission & Containment & Measure taken by the authority & Effects on daily activities \\
\hline \multicolumn{6}{|l|}{ Age (Years) } \\
\hline $20-30$ & $3.77 \pm 0.99$ & $4.10 \pm 0.99$ & $4.41 \pm 1.03$ & $3.54 \pm 0.86$ & $4.25 \pm 1.10$ \\
\hline $30-40$ & $3.66 \pm 0.96$ & $4.11 \pm 1.17$ & $5.04 \pm 1.80$ & $3.66 \pm 1.01$ & $4.27 \pm 1.15$ \\
\hline$>40$ & $3.74 \pm 0.94$ & $3.98 \pm 1.16$ & $4.66 \pm 1.03$ & $3.42 \pm 0.80$ & $4.33 \pm 1.05$ \\
\hline \multicolumn{6}{|l|}{ Gender } \\
\hline Male & $3.67 \pm 0.95$ & $3.98 \pm 1.05$ & $4.55 \pm 1.37$ & $3.47 \pm 0.84$ & $4.27 \pm 1.14$ \\
\hline Female & $3.90 \pm 1.00$ & $4.29 \pm 1.06$ & $4.72 \pm 0.98$ & $3.70 \pm 0.95$ & $4.27 \pm 0.99$ \\
\hline \multicolumn{6}{|l|}{ Occupation } \\
\hline Physician & $3.67 \pm 0.91$ & $4.17 \pm 1.06$ & $4.62 \pm 0.98$ & $3.37 \pm 0.71$ & $4.22 \pm 1.09$ \\
\hline Nurse & $3.76 \pm 1.06$ & $3.73 \pm 0.93$ & $4.60 \pm 2.00$ & $3.70 \pm 1.00$ & $4.30 \pm 1.15$ \\
\hline Pharmacist & $3.89 \pm 0.98$ & $4.39 \pm 1.10$ & $4.33 \pm 1.07$ & $3.80 \pm 1.00$ & $4.39 \pm 1.10$ \\
\hline Other HCPs & $3.76 \pm 0.99$ & $3.93 \pm 1.08$ & $4.83 \pm 1.05$ & $3.51 \pm 0.92$ & $4.27 \pm 1.07$ \\
\hline \multicolumn{6}{|c|}{ Experience (years) } \\
\hline \multicolumn{6}{|l|}{$<5$} \\
\hline $5-10$ & $3.75 \pm 0.98$ & $4.16 \pm 1.00$ & $4.48 \pm 0.99$ & $3.49 \pm 0.84$ & $4.24 \pm 1.12$ \\
\hline \multirow[t]{2}{*}{$>10$} & $3.84 \pm 1.00$ & $3.90 \pm 1.04$ & $5.10 \pm 2.10$ & $3.68 \pm 0.90$ & $4.26 \pm 1.18$ \\
\hline & $3.66 \pm 0.94$ & $4.00 \pm 1.17$ & $4.58 \pm 1.07$ & $3.57 \pm 0.95$ & $4.34 \pm 1.01$ \\
\hline \multicolumn{6}{|l|}{ Province } \\
\hline Aden & $3.73 \pm 1.00$ & $4.07 \pm 0.92$ & $4.68 \pm 1.00$ & $3.45 \pm 0.93$ & $4.48 \pm 1.15$ \\
\hline Hadramaut & $3.56 \pm 0.94$ & $3.86 \pm 1.17$ & $4.42 \pm 1.05$ & $3.48 \pm 0.83$ & $4.15 \pm 1.09$ \\
\hline Taiza & $3.88 \pm 0.91$ & $4.08 \pm 0.97$ & $4.54 \pm 0.97$ & $3.63 \pm 0.87$ & $4.06 \pm 1.01$ \\
\hline Aldhaleh & $3.33 \pm 0.70$ & $4.67 \pm 1.41$ & $4.44 \pm 1.24$ & $3.89 \pm 0.93$ & $5.00 \pm 0.71$ \\
\hline Sanaa & $4.75 \pm 0.96$ & $4.00 \pm 1.41$ & $5.25 \pm 0.96$ & $3.00 \pm 0.00$ & $3.75 \pm 0.96$ \\
\hline $\mathrm{lbb}$ & $3.50 \pm 0.75$ & $4.12 \pm 0.83$ & $4.25 \pm 0.89$ & $3.37 \pm 0.52$ & $4.12 \pm 1.12$ \\
\hline Abyan & $4.50 \pm 2.12$ & $4.50 \pm 2.12$ & $5.00 \pm 1.41$ & $4.50 \pm 2.12$ & $4.50 \pm 2.12$ \\
\hline Hajjah & 3.00 & 3.00 & 5.00 & 3.00 & 6.00 \\
\hline Lahij & $4.57 \pm 1.27$ & $5.00 \pm 1.00$ & $5.16 \pm 1.17$ & $4.14 \pm 1.46$ & $4.86 \pm 1.21$ \\
\hline Shabwah & $3.45 \pm 0.82$ & $4.00 \pm 1.00$ & $5.27 \pm 3.38$ & $3.27 \pm 0.47$ & $4.27 \pm 1.10$ \\
\hline \multicolumn{6}{|c|}{ Posting (Placement) } \\
\hline \multicolumn{6}{|l|}{ Quarantine } \\
\hline Isolation & $3.63 \pm 1.08$ & $3.78 \pm 1.01$ & $4.48 \pm 1.01$ & $3.55 \pm 0.80$ & $4.07 \pm 1.07$ \\
\hline ICU & $3.42 \pm 0.70$ & $4.02 \pm 1.12$ & $4.35 \pm 1.00$ & $3.63 \pm 0.87$ & $4.05 \pm 0.92$ \\
\hline \multirow[t]{2}{*}{ Other } & $4.00 \pm 1.23$ & $3.90 \pm 0.92$ & $4.59 \pm 1.05$ & $4.00 \pm 1.34$ & $4.36 \pm 1.33$ \\
\hline & $3.85 \pm 0.95$ & $4.20 \pm 1.07$ & $4.75 \pm 1.44$ & $3.41 \pm 0.75$ & $4.40 \pm 1.10$ \\
\hline \multicolumn{6}{|c|}{ Training (COVID-19) } \\
\hline \multicolumn{6}{|l|}{ Yes } \\
\hline \multirow[t]{2}{*}{ No } & $3.75 \pm 0.98$ & $3.93 \pm 1.06$ & $4.47 \pm 1.06$ & $3.62 \pm 0.90$ & $4.18 \pm 1.08$ \\
\hline & $3.73 \pm 0.96$ & $4.28 \pm 1.04$ & $4.80 \pm 1.49$ & $3.43 \pm 0.85$ & $4.41 \pm 1.10$ \\
\hline
\end{tabular}


Table 4 indicates the coping strategies embraced by the respondents amid COVID-19 pandemic. The religious coping strategy was reported highest in province Aldhaleh (3.33 \pm 0.87$)$ followed by acceptance (3.14 \pm 0.69$)$ in province Lahij, and humor $(3.00 \pm 1.41,3.00 \pm 0.82)$ in province Abyan and Lahij respectively, whereas it was the lowest for behavioral disengagement $(0.00 \pm 0.00)$ in province Abyan and active coping $(0.00)$ in province Hajjah.

Table 4 Coping Strategies Adopted by the Participants 


\begin{tabular}{|c|c|c|c|c|c|c|c|c|c|c|}
\hline Variables & $\begin{array}{l}\text { Self- } \\
\text { distraction }\end{array}$ & $\begin{array}{l}\text { Active } \\
\text { coping }\end{array}$ & Denial & $\begin{array}{l}\text { Substance } \\
\text { use }\end{array}$ & $\begin{array}{l}\text { Emotional } \\
\text { support }\end{array}$ & $\begin{array}{l}\text { Informational } \\
\text { support }\end{array}$ & $\begin{array}{l}\text { Behavioral } \\
\text { disengagement }\end{array}$ & Venting & $\begin{array}{l}\text { Positive } \\
\text { reframing }\end{array}$ & Planninç \\
\hline \multicolumn{11}{|l|}{ Age (Years) } \\
\hline $20-30$ & $2.28 \pm 1.04$ & $1.89 \pm 1.75$ & $2.26 \pm 1.98$ & $2.49 \pm 1.96$ & $1.50 \pm 1.75$ & $2.54 \pm 1.15$ & $1.63 \pm 1.66$ & $2.54 \pm 1.13$ & $1.77 \pm 1.63$ & $2.41 \pm 1.1$ \\
\hline $30-40$ & $2.45 \pm 1.02$ & $2.18 \pm 1.88$ & $2.18 \pm 1.78$ & $2.54 \pm 1.78$ & $1.45 \pm 1.62$ & $2.52 \pm 1.28$ & $1.68 \pm 1.88$ & $2.75 \pm 1.18$ & $1.73 \pm 1.47$ & $2.52 \pm 1.1$ \\
\hline$>40$ & $2.50 \pm 1.13$ & $1.95 \pm 1.77$ & $2.02 \pm 1.81$ & $2.52 \pm 1.99$ & $1.33 \pm 1.70$ & $2.50 \pm 0.99$ & $1.55 \pm 1.55$ & $2.36 \pm 1.03$ & $1.57 \pm 1.61$ & $2.51 \pm 0.9$ \\
\hline \multicolumn{11}{|l|}{ Gender } \\
\hline Male & $2.41 \pm 1.04$ & $1.87 \pm 1.77$ & $2.09 \pm 1.9$ & $2.46 \pm 2.00$ & $1.38 \pm 1.70$ & $2.40 \pm 1.15$ & $1.54 \pm 1.73$ & $2.46 \pm 1.14$ & $1.62 \pm 1.63$ & $2.38 \pm 1.1$ \\
\hline Female & $2.27 \pm 1.07$ & $2.19 \pm 1.79$ & $2.40 \pm 1.86$ & $2.40 \pm 1.86$ & $1.63 \pm 1.700$ & $2.81 \pm 1.08$ & $1.81 \pm 1.08$ & $2.74 \pm 1.07$ & $1.93 \pm 1.46$ & $2.64 \pm 1.0$ \\
\hline \multicolumn{11}{|l|}{ Occupation } \\
\hline Physician & $2.51 \pm 1.04$ & $1.93 \pm 1.80$ & $2.34 \pm 1.95$ & $2.16 \pm 1.78$ & $1.16 \pm 1.55$ & $2.44 \pm 1.09$ & $1.51 \pm 1.58$ & $2.48 \pm 1.13$ & $1.54 \pm 1.58$ & $2.44 \pm 1.0$ \\
\hline Nurse & $2.27 \pm 1.17$ & $2.30 \pm 1.61$ & $2.14 \pm 1.75$ & $2.78 \pm 1.94$ & $1.92 \pm 1.89$ & $2.86 \pm 1.20$ & $1.92 \pm 1.82$ & $2.94 \pm 1.25$ & $2.13 \pm 1.49$ & $2.62 \pm 1.0$ \\
\hline Pharmacist & $2.08 \pm 0.97$ & $1.75 \pm 1.90$ & $1.94 \pm 1.80$ & $2.61 \pm 2.09$ & $1.61 \pm 1.50$ & $2.55 \pm 1.16$ & $1.54 \pm 1.70$ & $2.19 \pm 1.04$ & $1.47 \pm 1.50$ & $2.08 \pm 0.9$ \\
\hline Other HCPs & $2.41 \pm 1.02$ & $1.93 \pm 1.78$ & $2.17 \pm 2.01$ & $2.88 \pm 1.95$ & $1.51 \pm 1.92$ & $2.36 \pm 1.16$ & $1.63 \pm 1.76$ & $2.63 \pm 0.99$ & $1.93 \pm 1.69$ & $2.68 \pm 1.3$ \\
\hline
\end{tabular}

\section{Experience}

(years)

$<5$

5-10

$2.31+1.04 \quad 1.83+1.77 \quad 2.82+1.93$

$2.32 \pm 1.01 \quad 2.16 \pm 1.82 \quad 2.00 \pm 1.86$

$2.45 \pm 1.89 \quad 1.34 \pm 1.61 \quad 2.84 \pm 1.13$

$1.56 \pm 1.64$

$2.47 \pm 1.09 \quad 1.65 \pm 1.51 \quad 2.41 \pm 1.1$

$>10$

$2.50 \pm 1.11$

$2.14 \pm 1.77 \quad 2.05 \pm 1.85$

$2.74 \pm 1.89 \quad 1.68 \pm 1.72 \quad 2.61 \pm 1.28$

$2.03 \pm 1.97$

$2.93 \pm 1.18 \quad 2.00 \pm 1.79$

$2.61 \pm 1.1$

Posting

Quarantine

Isolation

$2.15 \pm 1.03$

$2.03 \pm 1.87 \quad 2.00 \pm 1.66$

$2.48 \pm 1.99$

$1.57 \pm 1.88 \quad 2.57 \pm 1.11$

$1.52 \pm 1.57$

$2.48 \pm 1.13 \quad 1.70 \pm 1.62 \quad 2.47 \pm 1.0$

$I C U$

Other

$2.16 \pm 1.04$

$2.02 \pm 1.53$

$1.70 \pm 1.57$

$2.52 \pm 1.93 \quad 1.22 \pm 1.45$

$2.52 \pm 1.15$

$1.30 \pm 1.32$

$2.44 \pm 1.19 \quad 1.52 \pm 1.40$

$2.22 \pm 0.8$

CU

$2.50 \pm 1.10$

$2.14 \pm 1.91$

$2.18 \pm 2.01$

$2.46 \pm 1.83$

$1.46 \pm 1.52$

$2.35 \pm 1.17$

$1.74 \pm 1.57$

$2.23 \pm 0.92 \quad 1.19 \pm 1.38$

$2.51 \pm 1.1$

$2.05 \pm 2.18$

$2.86 \pm 1.08 \quad 2.14 \pm 1.78$

$2.59 \pm 1.3$

$1.57 \pm 1.69$

$2.63 \pm 1.18 \quad 1.62 \pm 1.66 \quad 2.47 \pm 1.1$

\section{Province}

Aden

Hadramaut

$2.48 \pm 1.05$

$1.89 \pm 1.84 \quad 2.45 \pm 2.02$

$2.39 \pm 1.93 \quad 1.34 \pm 1.68 \quad 2.52 \pm 1.07$

Taiza

Aldhaleh

Sanaa

$\mathrm{Ibb}$

Abyan

Hajjah

Lahij

Shabwah

\section{$2.32 \pm 1.03$}

$1.98 \pm 1.84$

$2.36 \pm 2.06$

$2.45 \pm 2.08$

$1.04 \pm 1.36$

$2.70 \pm 1.29$

$1.54 \pm 1.84$

$2.35 \pm 1.10$

$2.32 \pm 1.06$

$2.25 \pm 1.80$

$2.22+1.75$

$2.38 \pm 1.97$

$3.10 \pm 1.76$

$2.07 \pm 1.73$

$2.83 \pm 1.07$

$0.78 \pm 1.39$

$2.11 \pm 0.78$

$0.50 \pm 0.58 \quad 2.00 \pm 0.00$

$0.87 \pm 0.83 \quad 2.50 \pm 1.19$

$0.00 \pm 0.00$

$1.50 \pm 0.71$

$2.00 \pm 1.41$

$0.00 \pm 0.00$

$0.50 \pm 0.71$

$0.50 \pm 0.71$

$0.00 \quad 3.00$

$2.71 \pm 0.75$

$2.64 \pm 1.43$

$1.14 \pm 1.86$

3.00

1.00

1.00

$2.14 \pm 1.07$

$2.18 \pm 1.33$
$1.49 \pm 1.55$

$1.56 \pm 1.70$

$1.98 \pm 1.71$

$0.55 \pm 1.01$

$0.75 \pm 0.50$

$2.00 \pm 1.31$

$0.00 \pm 0.00$

1.00

$1.14 \pm 1.21$

$2.09 \pm 2.66$
$2.50 \pm 1.13 \quad 1.59 \pm 1.72 \quad 2.25 \pm 1.0$

$2.46 \pm 1.14 \quad 1.65 \pm 1.57 \quad 2.52 \pm 1.1$

$2.86 \pm 1.10 \quad 1.97 \pm 1.47 \quad 2.64 \pm 1.1$

$1.67 \pm 0.71 \quad 0.67 \pm 1.32 \quad 1.78 \pm 0.6$

$2.50 \pm 1.00 \quad 2.50 \pm 2.08 \quad 2.75 \pm 0.5$

$2.12 \pm 1.12 \quad 2.50 \pm 1.41 \quad 2.75 \pm 1.2$

$2.00 \pm 0.00 \quad 0.50 \pm 0.71 \quad 1.50 \pm 0.7$

$\begin{array}{lll}1.00 & 1.00 \quad 2.00\end{array}$

$2.43 \pm 0.79 \quad 1.00 \pm 1.41 \quad 2.33 \pm 0.5$

$2.82 \pm 1.33 \quad 2.00 \pm 1.73 \quad 2.45 \pm 1.3$

\section{Training}

(COVID-19)

Yes

No

$\begin{array}{lll}2.23 \pm 1.03 & 1.71 \pm 1.67 & 2.02 \pm 1.85 \\ 2.55 \pm 1.06 & 2.33 \pm 1.88 & 2.43 \pm 1.94\end{array}$

$2.27 \pm 1.91$

$1.41 \pm 1.80$

$2.35 \pm 1.11$

$1.59 \pm 1.74$

$2.45 \pm 1.12 \quad 1.53 \pm 1.52 \quad 2.32 \pm 1.0$

$2.55 \pm 1.06$

$.52 \pm 1.5$

$2.77 \pm 1.15$

$1.67 \pm 1.60$

$2.69 \pm 1.12 \quad 1.99 \pm 1.64 \quad 2.65 \pm 1.2$ 
Most of the coping strategies followed by the HCPs is vary from one province to another, in which maximum used strategy in most provinces in Yemen is faith-based religion, meanwhile, emotional support shows less strategy to be followed in many provinces.

Multiple comparisons of coping strategies among selected variables were illustrated in table $\mathbf{5}$. Bonferroni correction revealed no significant differences in coping style among age categories except in the age group of 20-30 years as compared to 30-40 years that was statistically significant in self-blame ( $p=$ 0.046) as a coping strategy. While comparing coping strategies occupation-wise, a significant relationship was demonstrated only in nurse versus pharmacist comparison on venting $(p=0.026)$. As compared to $>10$ years experienced HCPs, the HCPs with $5-10$ years of experience showed high significance on selfblame $(\mathrm{p}=0.034)$. Furthermore, no statistically significant difference was reported in comparing posting areas regarding coping strategies among all HCPs.

Table 5 Multiple Comparisons of Coping Strategies among Selected Variable (Bonferroni Correction) 


\begin{tabular}{|c|c|c|c|c|c|c|c|c|c|c|c|}
\hline Variables & $\begin{array}{l}\text { Self- } \\
\text { distraction }\end{array}$ & $\begin{array}{l}\text { Active } \\
\text { coping }\end{array}$ & Denial & $\begin{array}{l}\text { Substance } \\
\text { use }\end{array}$ & $\begin{array}{l}\text { Emotional } \\
\text { support }\end{array}$ & $\begin{array}{l}\text { Informational } \\
\text { support }\end{array}$ & $\begin{array}{l}\text { Behavioral } \\
\text { disengagement }\end{array}$ & Venting & $\begin{array}{l}\text { Positive } \\
\text { reframing }\end{array}$ & Planning & Humor \\
\hline \multicolumn{12}{|l|}{ Age (Years) } \\
\hline $\begin{array}{l}20-30 \mathrm{Vs} \\
30-40\end{array}$ & 1.00 & 1.00 & 1.00 & 1.00 & 1.00 & 1.00 & 1.00 & 0.892 & 1.00 & 1.00 & 1.00 \\
\hline \multirow{2}{*}{$\begin{array}{l}20-30 \mathrm{Vs} \\
>40\end{array}$} & 0.748 & 1.00 & 1.00 & 1.00 & 1.00 & 1.00 & 1.00 & 1.00 & 1.00 & 1.00 & 1.00 \\
\hline & 1.00 & 1.00 & 1.00 & 1.00 & 1.00 & 1.00 & 1.00 & 0.321 & 1.00 & 1.00 & 1.00 \\
\hline \multicolumn{12}{|l|}{$\begin{array}{l}30-40 \mathrm{Vs} \\
>40\end{array}$} \\
\hline \multicolumn{12}{|l|}{ Occupation } \\
\hline $\begin{array}{l}\text { Physician } \\
\text { Vs Nurse }\end{array}$ & 1.00 & 1.00 & 1.00 & 0.587 & 1.00 & 0.388 & 1.00 & 0.214 & 0.350 & 1.00 & 1.00 \\
\hline $\begin{array}{l}\text { Physician } \\
\text { Vs } \\
\text { Pharmacist }\end{array}$ & 0.270 & 1.00 & 1.00 & 1.00 & 1.00 & 1.00 & 1.00 & 1.00 & 1.00 & 0.628 & 0.782 \\
\hline \multirow{2}{*}{$\begin{array}{l}\text { Physician } \\
\text { Vs others }\end{array}$} & 1.00 & 1.00 & 1.00 & 0.294 & 0.143 & 1.00 & 1.00 & 1.00 & 1.00 & 1.00 & 1.00 \\
\hline & 1.00 & 1.00 & 1.00 & 1.00 & 1.00 & 1.00 & 1.00 & 0.026 & 0.442 & 0.218 & 1.00 \\
\hline \multicolumn{12}{|l|}{$\begin{array}{l}\text { Nurse Vs } \\
\text { Pharmacist }\end{array}$} \\
\hline \multirow{2}{*}{$\begin{array}{l}\text { Nurse Vs } \\
\text { Others }\end{array}$} & 1.00 & 1.00 & 1.00 & 1.00 & 1.00 & 0.311 & 1.00 & 1.00 & 1.00 & 1.00 & 1.00 \\
\hline & 1.00 & 1.00 & 1.00 & 1.00 & 1.00 & 1.00 & 1.00 & 0.507 & 1.00 & 0.102 & 1.00 \\
\hline \multicolumn{12}{|l|}{$\begin{array}{l}\text { Pharmacist } \\
\text { Vs Others }\end{array}$} \\
\hline \multicolumn{12}{|l|}{$\begin{array}{l}\text { Experience } \\
\text { (years) }\end{array}$} \\
\hline$<5$ Vs 5-10 & 1.00 & 1.00 & 1.00 & 1.00 & 0.981 & 1.00 & 0.505 & 0.130 & 0.857 & 1.00 & 1.00 \\
\hline$<5 \mathrm{Vs}>10$ & 0.816 & 0.845 & 1.00 & 1.00 & 1.00 & 1.00 & 1.00 & 1.00 & 1.00 & 1.00 & 1.00 \\
\hline $\begin{array}{l}5-10 \mathrm{Vs} \\
>10\end{array}$ & 1.00 & 1.00 & 1.00 & 1.00 & 1.00 & 1.00 & 0.518 & 0.216 & 1.00 & 1.00 & 1.00 \\
\hline \multicolumn{12}{|l|}{ Posting } \\
\hline $\begin{array}{l}\text { Quarantine } \\
\text { Vs } \\
\text { Isolation }\end{array}$ & 1.00 & 1.00 & 1.00 & 1.00 & 1.00 & 1.00 & 1.00 & 1.00 & 1.00 & 1.00 & 1.00 \\
\hline $\begin{array}{l}\text { Quarantine } \\
\text { Vs ICU }\end{array}$ & 1.00 & 1.00 & 1.00 & 1.00 & 0.190 & 1.00 & 0.757 & 1.00 & 1.00 & 1.00 & 0.647 \\
\hline $\begin{array}{l}\text { Quarantine } \\
\text { Vs Others }\end{array}$ & 0.897 & 1.00 & 1.00 & 1.00 & 1.00 & 1.00 & 1.00 & 1.00 & 1.00 & 1.00 & 1.00 \\
\hline $\begin{array}{l}\text { Isolation } \\
\text { Vs ICU }\end{array}$ & 1.00 & 1.00 & 1.00 & 1.00 & 0.419 & 0.379 & 1.00 & 0.195 & 1.00 & 1.00 & 1.00 \\
\hline $\begin{array}{l}\text { Isolation } \\
\text { Vs Others }\end{array}$ & 0.606 & 1.00 & 0.714 & 1.00 & 1.00 & 1.00 & 1.00 & 0.279 & 1.00 & 1.00 & 1.00 \\
\hline
\end{tabular}




\section{Discussion}

From the various studies regarding mental health issues of people around the globe amid such pandemic, there is no doubt that HCPs are experiencing unparalleled extents of COVID-19 related psychological stress across the personal and professional spheres. This is why, this study was undertaken to interpret the quality assessment of psychological exhaustion of COVID-19 pandemic in Yemeni HCPs as well as their behavior to such pandemic [16-20].

A total of $197 \mathrm{HCPs}$ from different provinces of the country participated in this study where a male to female ratio was found to be 2.18 with $68.5 \%$ male HCPs. Such findings of this study are in line with the study conducted in Nepal, where $54.2 \%$ of male participants were included [21]. Among the respondents, physicians were the majority HCPs whereas the nurses were found more vulnerable towards anxiety and depression. In gender-wise analysis, female HCPs were reported less in number as well as more depressed and anxious as compared to the male counterparts. This finding is in agreement with the outcome of a study conducted in Saudi Arabia by Al-Hanawi et al. (2020) [22]. The cultural perspective and the more concerns towards taking care of family members might be the contributing factors in this regard. More exposure towards the patients within the ward and workload are the leading cause of susceptibility of psychological stress among nurses [23].

Out of 22 provinces, the maximum number of HCPs participated from province Taiz (29.95\%) as it is the largely populated province of Yemen. Though it was a nationwide survey, no HCPs participated from 11 provinces because of inaccessibility in getting contacted due to COVID-19 pandemic and civil war effects.

Our finding of anxiety and depression in both gender were found to be of mild category which is in line with other previous studies such as studies conducted by Shechter et al. (2020) in New York and Salman et al. in Pakistan (2020) [24, 25].

The majority of HCPs (59\%) were already trained regarding COVID-19 management in our study. This finding contrasts with the previous result of Yemen where a majority of the respondents had never attended such kind of training [26]. Such finding possibly highlights the need to direct more attention toward developing educational courses and programmers related to COVID-19 [27]. Regarding the difference in depression with respect to age, experience, and working areas; our result did not reveal any significance, meanwhile, a significant statistical difference of depression score was reported in occupation-wise comparison between physician versus nurse $(p=0.017)$. This may be due to the more knowledge of the intensive effect of COVID-19 by the physician as compared to the nurse which was previously revealed by a study in Yemen [26].

our finding showed no difference in the anxiety of HCPs in working areas, which also contrasts with the previous studies in China that had revealed almost twice the risk of anxiety in different working areas [27]. Some studies have compared the mental disorders experienced by HCPs in areas where the pandemic was widely experienced compared to other regions. Anxiety, fear, and depression were much higher in HCPs in those areas because the HCPs working there are always more susceptible to infection [27].

A stressor such as health of self/family/loved-ones, transmission, containment, a measure taken by the authority, and effects on daily activities was found varying from province to province. The highest measures taken by the authority were in province eight meanwhile the status of the other remaining provinces was poor. This may be attributed to the poor health care facilities in Yemen to tackle such pandemic, where majorities of health care centers are not provided with the proper preventive facilities [28].

Our finding highlighted the importance to provide adequate psychological support to HCPs, as well as implement preventive measures to control the stressor among HCPs. Such findings correlate with the findings from Si et al. (2020) in China [4].

The general preparedness and capability to tackle COVID-19 were reported very poor by the majority of HCPs in our study, which corresponds with the various studies conducted in Yemen by different researchers that demonstrated the fragile healthcare system of Yemen and difficulty in coping with the scenario by HCPs working therein [28-30].

Comparing coping behaviors in Yemen HCPs towards COVID-19, a significant association was notified between the level of performance of participants and their occupation $(p=0.023$, which is in line with our finding which shows a significant relationship in the only nurse versus pharmacist comparison on venting $(p=0.026)$, meanwhile, the years of work experience showed no significance in our finding which contrasts the previous finding in Yemen $(p=0.011) 19$.

Eisenberg et al. (2012) had described two major elements of the coping strategies, namely "avoidant coping" and "approach coping" [31]. Avoidant coping was designated by the subscales of denial, behavioral disengagement, substance use, self-blame, venting, and self-distraction. Besides other subscales, religion and humor were regarded as adaptive coping. Similarly, approach coping was described by the subscales of active coping, positive reframing, acceptance, planning, informational support, and seeking emotional. Based on the avoidant, approach, and adaptive coping strategies; we assessed the type of wellness resources as a coping strategy in such COVID-19 adversity. We found the most respondents scored is adaptive coping based on faith-based religion, which is in contrast with a study conducted in New York by Shechter [24].

There were certain limitations in our study. Firstly, very few HCPs as respondents were available from some provinces due to the adverse scenario caused by COVID-19 as well as by the internal catastrophe of conflict. Secondly, the inherent selection bias cannot be ignored due to the exploratory kind of study. However, our finding has investigated the psychological impact, source of stress, and coping strategies of HCPs on COVID-19 from different health care institutions from various provinces of Yemen. 


\section{Conclusion}

This study investigated the level of anxiety, stress, depression, and the coping behavior thereof in healthcare professionals in Yemen. COVID-19 pandemic has caused a mild impact on the mental health status of Yemeni healthcare professionals. The most frequently adopted coping strategies in most provinces in Yemen were found faith-based religion. The trained professionals demonstrated less level of psychological stress. Our findings indicate the requirement of adequate plans and policies from administrative to clinical and welfare viewpoint in preparedness and preventive behaviors from the regulatory body that alleviates the psychological stress of such professionals for the efficient provision of better healthcare services throughout the nation.

\section{Abbreviations}

$\begin{array}{ll}\text { ANOVA: } & \text { Analysis of variance } \\ \text { COVID-19: } & \text { Coronavirus disease-19 } \\ \text { GAD-7: } & \text { Generalized anxiety disorder-7 } \\ \text { HCPs: } & \text { Healthcare professionals } \\ \text { ICU: } & \text { Intensive care unit } \\ \text { IHR: } & \text { Public Health Emergency of International Concern } \\ \text { PHEIC: } & \text { Patient health questionnaire-9 } \\ \text { PHQ-9: } & \text { Personal protective equipment } \\ \text { PPE: } & \text { Standard deviation } \\ \text { SD: } & \text { Statistical package for social sciences } \\ \text { SPSS: } & \text { World Health Organization } \\ \text { WHO: } & \end{array}$

\section{Declarations}

\section{Ethics approval and consent to participate}

The research has been performed in accordance with the Declaration of Helsinki. The ethics approval was granted by the Humans Ethics Committee (HEC), University of the Punjab, Lahore. Informed consent to participate in the study was obtained from participants.

\section{Consent for publication}

Not applicable

\section{Availability of data and materials}

The datasets used and/or analyzed during the current study will be available from the corresponding author on reasonable request.

\section{Competing interests}

The authors declare no competing conflict of interest.

\section{Funding}

No funding was obtained from any source.

\section{Authors' contributions}

NMAM: Conceptualization, Methodology, Data collection, Data curation, and Writing - Original draft preparation. MS: Conceptualization, Resources, Supervision, and Writing-Reviewing and editing. SK: Methodology, Formal analysis, and Writing- Reviewing and editing. MAAMA: Data collection, Visualization, and Writing- original draft preparation. MMSSK: Data collection, Visualization, and Writing- original draft preparation. MAJA: Data curation, Formal analysis, and Validation.

\section{Acknowledgements}

We appreciate the help of those healthcare professionals who participated as respondents in this study.

\section{Authors' information}

Page $12 / 14$ 


\section{Nagd Mohammed Ahmed Mahmood, PharmD, MPhil}

Palsm Pharmacy, Aden, Yemen

Punjab University College of Pharmacy, University of the Punjab, Lahore, Pakistan

nagdmahmood@gmail.com

\section{Mohammad Saleem, PhD}

Punjab University College of Pharmacy, University of the Punjab, Lahore, Pakistan

saleem2978@hotmail.com

\section{Sitaram Khadka, PharmD, MPhil}

Punjab University College of Pharmacy, University of the Punjab, Lahore, Pakistan

Shree Birendra Hospital; Nepalese Army Institute of Health Sciences, Kathmandu, Nepal

sitaram.khadka@naihs.edu.np

\section{Maroa Ahmed Ali Mohammed Alkamel}

Faculty of Medicine and Health Science, Taiz University, Taiz, Yemen

marwaalkamel1@gmail.com

\section{Maged Mohammed Salem Seed Khudhiere}

Faculty of Dentistry, University of Aden, Aden, Yemen

Health and Nutrition Foundation for Development (HAND), Aden, Yemen

maged.saeed52@gmail.com

\section{Muhammad Abdul Jabar Adnan}

Department of Sports Sciences and Physical Education, University of the Punjab, Lahore, Pakistan

abduljabar_4@yahoo.com

\section{References}

1. Jee Y. WHO International Health Regulations Emergency Committee for the COVID-19 outbreak. Epidemiol Health. 2020;42:e2020013.

2. Cucinotta D, Vanelli M. WHO Declares COVID-19 a Pandemic. Acta Biomed. 2020 Mar;91(1):157-60.

3. Lai J, Ma S, Wang Y, Cai Z, Hu J, Wei N, et al. Factors Associated With Mental Health Outcomes Among Health Care Workers Exposed to Coronavirus Disease 2019. JAMA Netw open. 2020 Mar;3(3):e203976.

4. Si M-Y, Su X-Y, Jiang Y, Wang W-J, Gu X-F, Ma L, et al. Psychological impact of COVID-19 on medical care workers in China. Infect Dis poverty. 2020 Aug;9(1):113.

5. Wilder-Smith A, Freedman DO. Isolation, quarantine, social distancing and community containment: pivotal role for old-style public health measures in the novel coronavirus (2019-nCoV) outbreak. J Travel Med. 2020 Mar;27(2).

6. Shigemura J, Ursano RJ, Morganstein JC, Kurosawa M, Benedek DM. Public responses to the novel 2019 coronavirus (2019-nCoV) in Japan: Mental health consequences and target populations. Vol. 74, Psychiatry and clinical neurosciences. 2020. p. 281-2.

7. Torales J, O'Higgins M, Castaldelli-Maia JM, Ventriglio A. The outbreak of COVID-19 coronavirus and its impact on global mental health. Int J Soc Psychiatry. 2020 Jun;66(4):317-20.

8. Huang JZ, Han MF, Luo TD, Ren AK, Zhou XP. [Mental health survey of medical staff in a tertiary infectious disease hospital for COVID-19]. Zhonghua lao dong wei sheng zhi ye bing za zhi = Zhonghua laodong weisheng zhiyebing zazhi = Chinese J Ind Hyg Occup Dis. 2020 Mar;38(3):192-5.

9. Kang L, Li Y, Hu S, Chen M, Yang C, Yang BX, et al. The mental health of medical workers in Wuhan, China dealing with the 2019 novel coronavirus. Vol. 7 , The lancet. Psychiatry. 2020. p. e14.

10. Xiang Y-T, Yang Y, Li W, Zhang L, Zhang Q, Cheung T, et al. Timely mental health care for the 2019 novel coronavirus outbreak is urgently needed. The lancet Psychiatry. 2020 Mar;7(3):228-9.

11. Al-Subari S, Khadka S, Mahmood N, Alhouzani T, Shrestha D. COVID-19 Pandemic in Yemen. J Karnali Acad Heal Sci. 2020;3(2):159-63.

12. Spitzer RL, Kroenke K, Williams JBW, Löwe B. A brief measure for assessing generalized anxiety disorder: the GAD-7. Arch Intern Med. 2006 May;166(10):1092-7. 
13. Kroenke K, Spitzer RL, Williams JB. The PHQ-9: validity of a brief depression severity measure. J Gen Intern Med. 2001 Sep;16(9):606-13.

14. Wong TW, Gao Y, Tam WWS. Anxiety among university students during the SARS epidemic in Hong Kong. Vol. 23, Stress and Health. 2007. p. 31-5.

15. Carver CS. You want to measure coping but your protocol's too long: consider the brief COPE. Int J Behav Med. 1997;4(1):92-100.

16. Stuijfzand S, Deforges C, Sandoz V, Sajin C-T, Jaques C, Elmers J, et al. Psychological impact of an epidemic/pandemic on the mental health of healthcare professionals: a rapid review. BMC Public Health. 2020 Aug;20(1):1230.

17. Lu W, Wang H, Lin Y, Li L. Psychological status of medical workforce during the COVID-19 pandemic: A cross-sectional study. Psychiatry Res. 2020 Jun;288:112936.

18. Raudenská J, Steinerová V, Javůrková A, Urits I, Kaye AD, Viswanath 0 , et al. Occupational burnout syndrome and post-traumatic stress among healthcare professionals during the novel coronavirus disease 2019 (COVID-19) pandemic. Best Pract Res Clin Anaesthesiol. 2020 Sep;34(3):553-60.

19. Ghosh R, Dubey MJ, Chatterjee S, Dubey S. Impact of COVID -19 on children: special focus on the psychosocial aspect. Minerva Pediatr. 2020 Jun;72(3):226-35.

20. Chew QH, Wei KC, Vasoo S, Chua HC, Sim K. Narrative synthesis of psychological and coping responses towards emerging infectious disease outbreaks in the general population: practical considerations for the COVID-19 pandemic. Singapore Med J. 2020 Jul;61(7):350-6.

21. Sigdel A, Bista A, Bhattarai N, Pun BC, Giri G, Marqusee H, et al. Depression, Anxiety and Depression-anxiety comorbidity amid COVID-19 Pandemic: An online survey conducted during lockdown in Nepal. medRxiv [Internet]. 2020; Available from: https://www.medrxiv.org/content/early/2020/05/06/2020.04.30.20086926

22. Al-Hanawi MK, Mwale ML, Alshareef N, Qattan AMN, Angawi K, Almubark R, et al. Psychological Distress Amongst Health Workers and the General Public During the COVID-19 Pandemic in Saudi Arabia. Risk Manag Healthc Policy. 2020;13:733-42.

23. Maunder RG, Lancee WJ, Rourke S, Hunter JJ, Goldbloom D, Balderson K, et al. Factors associated with the psychological impact of severe acute respiratory syndrome on nurses and other hospital workers in Toronto. Psychosom Med. 2004;66(6):938-42.

24. Shechter A, Diaz F, Moise N, Anstey DE, Ye S, Agarwal S, et al. Psychological distress, coping behaviors, and preferences for support among New York healthcare workers during the COVID-19 pandemic. Gen Hosp Psychiatry [Internet]. 2020;66:1-8. Available from:

https://www.sciencedirect.com/science/article/pii/S0163834320300839

25. Salman M, Raza MH, Ul Mustafa Z, Khan TM, Asif N, Tahir H, et al. The psychological effects of COVID-19 on frontline healthcare workers and how they are coping: a web-based, cross-sectional study from Pakistan. medRxiv [Internet]. 2020; Available from: https://www.medrxiv.org/content/early/2020/06/05/2020.06.03.20119867

26. Alrubaiee GG, Al-Qalah TAH, Al-Aawar MSA. Knowledge, attitudes, anxiety, and preventive behaviours towards COVID-19 among health care providers in Yemen: an online cross-sectional survey. BMC Public Health. 2020 Oct;20(1):1541.

27. Al Nsour M, Khader Y, Al Serouri A, Bashier H, Osman S. Awareness and Preparedness of Field Epidemiology Training Program Graduates to Respond to COVID-19 in the Eastern Mediterranean Region: Cross-Sectional Study. JMIR Med Educ [Internet]. 2020 Sep;6(1):e19047. Available from:

https://doi.org/10.2196/19047

28. Zawiah M, Al-Ashwal FY, Saeed RM, Kubas M, Saeed S, Khan AH, et al. Assessment of Healthcare System Capabilities and Preparedness in Yemen to Confront the Novel Coronavirus 2019 (COVID-19) Outbreak: A Perspective of Healthcare Workers. Front public Heal. 2020;8:419.

29. Dureab F, Al-Awlaqi S JA. COVID-19 in Yemen: preparedness measures in a fragile state. Lancet Public Heal. 2020;

30. WHO. Yemen: Health Systems Profile [Internet]. 2013. Available from: https://applications.emro.who.int/docs/Country_profile_2013_EN_15404.pdf?f

31. Eisenberg SA, Shen B-J, Schwarz ER, Mallon S. Avoidant coping moderates the association between anxiety and patient-rated physical functioning in heart failure patients. J Behav Med. 2012 Jun;35(3):253-61.

\section{Supplementary Files}

This is a list of supplementary files associated with this preprint. Click to download.

- QuestionnareEnglish.docx

- QuestionnaireArabic.docx 